\title{
Return dynamics of index-linked bond portfolios
}

\author{
Matti Koivu* Teemu Pennanen ${ }^{\dagger}$
}

June 19, 2013

\begin{abstract}
Bond returns are known to exhibit mean reversion, autocorrelation and other dynamic properties that differentiate them from e.g. stock returns. Index-linked bonds bring in further characteristics that complicate the modeling of returns over time. Such models are essential, however, in strategic portfolio analysis and quantitative risk management. This paper shows that the modeling of index-linked bond portfolios can be reduced to statistical modeling of the portfolio's yield to maturity and the underlying index. For these quantities, many well established models already exist. Using historical data from 12 different markets, we show that the two risk factors explain consistently over $97 \%$ of monthly return variations over the past decade including the recent financial crisis.
\end{abstract}

\section{Introduction}

Bond portfolios are subject to a large number of risk factors. Even in the case of fixed rate government bonds, portfolio returns depend in general on the whole yield curve. Bond returns can also be described in terms of yield to maturity, which reduces return uncertainty into a single portfolio-specific factor. Such simple descriptions are easy to understand and to model, which makes them useful in portfolio analysis and risk management; see e.g. Fabozzi [2010, 2011]. In particular, the connection between bond returns and yields explains many empirically observed properties of bond return dynamics. For example, mean reversion and autocorrelation properties of bond returns can be traced back to time series properties of interest rates.

This paper shows that index-linked bonds allow for equally simple descriptions in terms of the yield to maturity and the underlying index. The two risk factors are found to explain consistently over $97 \%$ of monthly return variations on inflation-linked bonds issued by Canada, France, South Africa, Sweden, UK and the US. For government bonds, which correspond to a constant index, the explanatory power of our model is close to $100 \%$.

\footnotetext{
* Nordic Investment Bank

$\dagger$ Department of Mathematics, King's College London, teemu. pennanen@kcl.ac.uk, corresponding author
} 
The models presented in this paper are particularly useful in dynamic portfolio analysis and risk management where dynamic statistical models of portfolio returns are required. This paper reduces the task of return modeling to the statistical modeling of the yield and the underlying index for which many statistically validated models are available. Computational applications of the models described in this paper can be found in Hilli et al. [2011a,b].

\section{Returns on bond portfolios}

Consider a bond or a portfolio of bonds with outstanding payments at times $t_{1}<t_{2}<\cdots<t_{N}$. We assume that the payments are linked to an underlying index $I$ so that the payment at time $t_{n}$ will be $c_{n} I_{t_{n}}$ where $c_{n}$ is a given nominal payment. The portfolio's yield to maturity (or internal rate of return) $Y_{t}$ at time $t<t_{1}$ is defined as the solution of the equation

$$
P_{t}=\sum_{n=1}^{N} e^{-Y_{t}\left(t_{n}-t\right)} c_{n} I_{t},
$$

where $P_{t}$ is the portfolio's market price and $c_{n} I_{t}$ are the nominal payments outstanding at time $t$. In the case of inflation-linked bonds where $I$ is the consumer price index, $Y$ is known as the real yield. In survivor bonds, the underlying index $I$ is defined as the fraction of survivors in a given population. Such bonds have attracted a lot of attention in the recent literature on longevity risk management; see e.g. Biffis and Blake [2010]. The index $I$ could also be used to model default losses in the presence of counterparty risk.

The anticipated development of the index $I$ affects the market price of the portfolio and thus also its yield to maturity. In the case of inflation-linked bonds, the amount $c_{n} I_{t_{n}}$ that will be received at time $t_{n}$ is usually expected to be higher than the nominal payment $c_{n} I_{t}$ at time $t$. This is reflected as a higher price and lower yield than for a fixed rate bond with the same nominal payment structure. In the case of defaultable bonds, on the other hand, the realized payments $c_{n} I_{t_{n}}$ are less than or equal to outstanding payments $c_{n} I_{t}$ so the price is typically lower and the yield higher than for a corresponding default-free bond, hence the credit spread.

Our aim is to describe bond portfolio returns in terms of time, the yield and the underlying index without complete knowledge of the outstanding payments. This will allow us to describe statistical properties of bond returns in terms of the yield and the index whose behavior is well understood. Our strategy is to apply Ito's lemma to the logarithmic price index. Having defined the yield through (1), we can express the market price (much as in [Fabozzi, 2011, Chapter 42]) as $P_{t}=P\left(t, Y_{t}, I_{t}\right)$ where the function $P$ is defined by

$$
P(t, Y, I)=\sum_{n=1}^{N} e^{-Y\left(t_{n}-t\right)} c_{n} I .
$$


The first order derivatives of the logarithmic price are

$$
\frac{\partial \ln P}{\partial t}=Y, \quad \frac{\partial \ln P}{\partial Y}=-D, \quad \frac{\partial \ln P}{\partial I}=\frac{1}{I},
$$

where

$$
D=\frac{1}{P_{t}} \sum_{n=1}^{N}\left(t_{n}-t\right) e^{-Y\left(t_{n}-t\right)} c_{n} I_{t}
$$

is the duration of the portfolio. Denoting the convexity by

$$
C=\frac{1}{P} \frac{\partial^{2} P_{t}}{\partial Y^{2}}=\frac{1}{P} \sum_{n=1}^{N}\left(t_{n}-t\right)^{2} e^{-Y\left(t_{n}-t\right)} c_{n} I_{t},
$$

we get

$$
\frac{\partial^{2} \ln P}{\partial t \partial Y}=1, \quad \frac{\partial^{2} \ln P}{\partial Y^{2}}=C-D^{2}, \quad \frac{\partial^{2} \ln P}{\partial I^{2}}=-\frac{1}{I^{2}}
$$

while all other second-order derivatives of the log-price are zero. Applying Ito's lemma to the log-price with respect to the three dimensional process $(t, Y, I)$ gives $^{1}$

$$
d \ln P=Y d t-D d Y+\frac{1}{I} d I+\frac{1}{2} d[t, Y]+\frac{1}{2}\left(C-D^{2}\right) d[Y]-\frac{1}{2} \frac{1}{I^{2}} d[I],
$$

where $[Y]$ and $[I]$ denote the quadratic variation of the yield and the index, respectively, and $[t, Y]$ the covariation of time and the yield. When $Y$ is continuous, $[t, Y]=0$. By Ito's lemma again,

$$
d \ln I=\frac{1}{I} d I-\frac{1}{2} \frac{1}{I^{2}} d[I]
$$

so equation (3) simplifies to

$$
d \ln P_{t}=Y_{t} d t-D_{t} d Y_{t}+d \ln I_{t}+\frac{1}{2}\left(C_{t}-D_{t}^{2}\right) d[Y]_{t} .
$$

This formula describes the price dynamics in terms of time $t$, the yield $Y$ and the index $I$. The payment structure $\left(c_{n}, t_{n}\right), n=1, \ldots, N$ affects the price dynamics only through duration and convexity. In a portfolio that is regularly updated to maintain a fixed duration and convexity, the main return uncertainty comes from the development of the yield and the index. Formula (4) extends the analysis of Jarrow [1978] by allowing for index-linkage and by relaxing the assumption that the yield follows a geometric Brownian motion. Indeed, if $Y$ is an Ito-process with $d Y_{t}=\mu_{t} d t+\sigma_{t} d W_{t}$, where $W$ is a Brownian motion, we have $d[Y]_{t}=\sigma_{t} d t$.

Formula (4) clearly differentiates return characteristics of bond indices from those of stock indices. The first and the last term in (4) are always nonnegative.

\footnotetext{
${ }^{1}$ It is enough to assume that $Y$ and $I$ are continuous semimartingales; see e.g. [Kallenberg, 2002, Theorem 17.18].
} 
On the other hand, if the yield is unbounded from above, the term $-D d Y$ makes the distribution of the log-return unbounded from below. The first term in formula (4) explains the autocorrelations of bond returns observed in the empirical analysis of e.g. Reilly et al. [1992]. Indeed, yields are known to exhibit mean-reversion and autocorrelation (see e.g. Aït-Sahalia [1996]) so the term $Y_{t} d t$ in formula (4) carries these properties also to the returns. Shorter the maturity, smaller the duration and convexity and thus, stronger the relative contribution of the first term. This is confirmed by the empirical analysis of Reilly et al. [1992] who find that autocorrelation is highest in shorter maturities. When maturity approaches zero, the duration and convexity approach zero as well and formula (4) simplifies to $d \ln P_{t}=Y_{t} d t+d \ln I_{t}$.

\section{Returns on regularly rebalanced portfolios}

Assume now that the bond portfolio is regularly rebalanced to maintain constant duration and convexity. Many publicly available bond-indices are periodically rebalanced so that these characteristics remain approximately constant. Integrating both sides of (4) over a given holding period $[s, t]$ we then get

$$
\Delta \ln P=\int_{s}^{t} Y_{u} d u-D \Delta Y+\Delta \ln I+\frac{1}{2}\left(C-D^{2}\right) \Delta[Y],
$$

where $\Delta Y=Y_{t}-Y_{s}, \Delta \ln I=\ln I_{t}-\ln I_{s}$ and $\Delta[Y]=[Y]_{t}-[Y]_{s}$. In particular, return on unit investment is then given by

$$
\frac{P_{t}}{P_{s}}=\exp \left(\int_{s}^{t} Y_{u} d u-D \Delta Y+\frac{1}{2}\left(C-D^{2}\right) \Delta[Y]\right) \frac{I_{t}}{I_{s}} .
$$

The above suggests that the log-return can be approximated by

$$
\Delta \ln P \approx \frac{Y_{s}+Y_{t}}{2} \Delta t-D \Delta Y+\Delta \ln I+\frac{1}{2}\left(C-D^{2}\right)(\Delta Y)^{2} .
$$

This is, in fact, nothing but a second order Taylor-approximation of the logarithm of (2). Related formulas have been derived in Christensen and Sørensen [1994] and Chance and Jordan [1996]. While Christensen and Sørensen [1994] analyzed price sensitivities with respect to a general level of interest rates, we have focused explicitly on a given portfolio and its yield to maturity. Chance and Jordan [1996] developed second order approximations of bond returns in absolute terms rather than relative terms as above. This resulted in more complicated formulas with five different terms for the effects of time and yield changes alone. Neither Christensen and Sørensen [1994] nor Chance and Jordan [1996] considered index-linked bonds. 


\subsection{Fixed rate government bonds}

In the case of fixed rate default-free bonds, the underlying index $I$ is constant so the return formula (5) becomes

$$
\Delta \ln P \approx \frac{Y_{s}+Y_{t}}{2} \Delta t-D \Delta Y+\frac{1}{2}\left(C-D^{2}\right)(\Delta Y)^{2} .
$$

This is similar to the model studied e.g. in Ilmanen [1992] but there the time component was ignored and the approximation was based on a Taylor-approximation of the price itself instead of its logarithm.

Our data set covers end of month observations of the Barclays' market capitalization weighted total return indices, durations and yields for France, Germany, Italy, United Kingdom, United States and the Euro area ${ }^{2}$. The length of the time series for each country is given at the bottom of Table 1.

We fit the following two models to the data

$$
\begin{array}{ll}
\text { Model 1: } & \Delta \ln P_{s} \approx c+\frac{Y_{s}+Y_{t}}{2} \Delta t-D \Delta Y_{s}, \\
\text { Model 2: } & \Delta \ln P_{s} \approx c+\frac{Y_{s}+Y_{t}}{2} \Delta t-D \Delta Y_{s}+\gamma\left(\Delta Y_{s}\right)^{2} .
\end{array}
$$

The parameters $c, D$ and $\gamma=\frac{1}{2}\left(C-D^{2}\right)$ are estimated by ordinary least squares. Table 1 displays the estimation results for the two models. Model 1 already provides an almost perfect fit to the total return data with $R^{2}$ values ranging from $99.6 \%$ to $99.8 \%$. The fit has been good consistently over time and the residuals have remained marginal even during the recent financial crisis. The $R^{2}$ values being close to $100 \%$ there is not much room for improvement when adding the second-order term. The estimated coefficients $\gamma$ in Model 2 are generally significant at a $5 \%$ level but, consistently with the findings of Chance and Jordan [1996], the improvements in the $R^{2}$ statistics are marginal, less than $0.02 \%$ points in all the studied markets. The Partial- $R^{2}$ statistic is defined as the $R^{2}$-statistic obtained by regressing the residual of Model 1 with the quadratic term. The quadratic term of Model 2 explains less than $5.11 \%$ of the residual variance of Model 1 . The estimated constant $c$ in Model 1 deviates from zero at $5 \%$ significance level, but with varying signs. This may indicate that the model has not entirely captured all the systematic return components. The addition of the quadratic term in Model 2 mitigates this effect to some extent.

Our results are consistent with Ilmanen [1992] who finds that the explanatory power of duration has increased over time. In his empirical study, Ilmanen [1992] found that the duration explained $80 \%$ to $90 \%$ of return variance of fixed rate government bonds during the 1980s. The nearly $100 \% R^{2}$-values for Model 1 during the past decade suggest that the trend has continued. It should be noted, however, that our results are not directly comparable since our model explains the log-returns and it includes the time component.

\footnotetext{
${ }^{2}$ Further information is available online at https://ecommerce.barcap.com/indices/index.dxml
} 
Table 1: Regression statistics for fixed-rate government bonds

\begin{tabular}{r|cccccc}
\hline & FRA & GER & IT & UK & US & EURO \\
\hline Model 1 & & & & & & \\
$100 * c$ & 0.0147 & 0.0083 & 0.0084 & -0.0195 & -0.0202 & 0.015 \\
$R^{2}$ & $(3.5842)$ & $(1.7215)$ & $(1.9339)$ & $(-4.8403)$ & $(-3.0658)$ & $(4.1467)$ \\
Model 2 & $99.76 \%$ & $99.64 \%$ & $99.68 \%$ & $99.84 \%$ & $99.71 \%$ & $99.79 \%$ \\
$100 * c$ & 0.007 & 0.0007 & -0.0001 & -0.0256 & -0.0277 & 0.01 \\
& $(1.4255)$ & $(0.1186)$ & $(-0.0178)$ & $(-5.7509)$ & $(-3.6689)$ & $(2.2687)$ \\
$\gamma$ & 24.4889 & 24.1545 & 32.5155 & 4.8396 & 11.1262 & 17.031 \\
$R^{2}$ & $(2.7949)$ & $(2.2625)$ & $(2.5933)$ & $(3.0699)$ & $(1.9703)$ & $(1.9131)$ \\
\hline Partial- $R^{2}$ & $59.77 \%$ & $99.65 \%$ & $99.70 \%$ & $99.85 \%$ & $99.72 \%$ & $99.79 \%$ \\
\hline Data start & $1997-12$ & $3.41 \%$ & $4.43 \%$ & $2.63 \%$ & $2.84 \%$ & $2.46 \%$ \\
Data end & $2010-03$ & $2010-03$ & $2010-03$ & $2010-03$ & $2010-03$ & $2010-03$ \\
\hline
\end{tabular}

Notes. The table contains estimation results for the models:

Model 1: $\Delta \ln P_{s} \approx c+Y_{s} \Delta t-D_{t} \Delta Y_{s}$,

Model 2: $\Delta \ln P_{s} \approx c+Y_{s} \Delta t-D_{t} \Delta Y_{s}+\gamma\left(\Delta Y_{s}\right)^{2}$,

Numbers in parentheses are the $t$-statistics for the estimated coefficients.

Partial- $R^{2}$ is defined as the $R^{2}$-statistic obtained by regressing the residual of

Model 1 with the quadratic term.

\section{$3.2 \quad$ Inflation-linked bonds}

In inflation-linked bonds, principal and coupon payments are tied to the consumer price index. More precisely, the underlying index $I_{t}$ at time $t$ is defined as the consumer price index reported for some time before $t$. The time-lag is known as the indexation lag. We refer the reader to [Deacon et al., 2004, Chapter 2] for more details on inflation-linked bonds.

For inflation-linked bonds, (5) can be written as

$$
\Delta \ln P \approx \frac{Y_{s}+Y_{t}}{2} \Delta t-D \Delta Y+\pi_{t} \Delta t+\frac{1}{2}\left(C-D^{2}\right)(\Delta Y)^{2},
$$

where $\pi_{t}$ is the annualized rate of inflation over the period $[t-\delta, s-\delta]$ where $\delta$ denotes the indexation lag. To evaluate the significance of the underlying index, we consider two model specifications

$$
\begin{array}{ll}
\text { Model 1: } & \Delta \ln P_{s} \approx c+Y_{s} \Delta t-D_{t} \Delta Y_{s}, \\
\text { Model 2: } & \Delta \ln P_{s} \approx c+\left(Y_{s}+\pi_{s}\right) \Delta t-D_{t} \Delta Y_{s},
\end{array}
$$

where the first one ignores the third term in (7).

We use monthly observations of Barclays inflation-linked government bond index data. The data consists of total return indices, yields and durations on portfolios of inflation-linked government bonds for Canada, France, South Africa, Sweden, United Kingdom and United States ${ }^{3}$. The bonds' cash flows arelinked to the evolution of country specific inflation indices (usually the general

\footnotetext{
${ }^{3}$ Further information is available online at https://ecommerce.barcap.com/indices/index.dxml
} 
consumer price index). The monthly time series of appropriate inflation indices were obtained from Eurostat and the national statistical authorities' websites. Since the indexation lag is not specified in the data, we select the lag $\delta$ that gives the best fit to historical data. In all the studied countries, the data strongly supports one specific choice of the lag. The lags are given in Table 2 along with the regression statistics for the two models in the six markets.

The inclusion of the inflation term in Model 2 results in a substantial improvement over Model 1. The reduction in the residuals between Model 2 and Model 1 is caused solely by the incorporation of the inflation term. The Partial$R^{2}$ statistic gives the $R^{2}$-statistic for regressing the residual of Model 1 with the inflation term. The fact that the estimated constant terms in Model 2 are generally insignificant is another indication that the model is well specified. The lower $R^{2}$ value for South Africa is mainly caused by one outlier observation caused by pricing distortions in the local conventional bond market in 2002 and the subsequent inversion of the break-even inflation curve.

Table 2: Regression statistics for inflation-indexed government bonds

\begin{tabular}{r|cccccc}
\hline & CAN & FRA & SA & SWE & UK & US \\
\hline Model 1 & & & & & & \\
100*c $^{*}$ & 0.1642 & 0.1328 & 0.4935 & 0.1114 & 0.2073 & 0.1966 \\
t-stat & $(6.201)$ & $(5.544)$ & $(9.367)$ & $(3.678)$ & $(7.611)$ & $(5.888)$ \\
$R^{2}$ & $97.48 \%$ & $95.46 \%$ & $78.6 \%$ & $91.29 \%$ & $96.06 \%$ & $94.41 \%$ \\
Model 2 & & & & & & \\
100*c $^{*}$ & 0.0046 & -0.0098 & -0.0069 & 0.0087 & -0.0151 & -0.0004 \\
t-stat & $(0.628)$ & $(-0.839)$ & $(-0.389)$ & $(1.465)$ & $(-1.918)$ & $(-0.066)$ \\
$R^{2}$ & $99.80 \%$ & $98.91 \%$ & $97.58 \%$ & $99.66 \%$ & $99.67 \%$ & $99.82 \%$ \\
\hline Partial- $R^{2}$ & $92.22 \%$ & $76.02 \%$ & $88.69 \%$ & $96.14 \%$ & $91.43 \%$ & $96.78 \%$ \\
Indexation-lag & 2 & 2 & 3 & 2 & 1 & 3 \\
\hline Data start & $1996-12$ & $1998-09$ & $2000-03$ & $1996-12$ & $1996-01$ & $1997-03$ \\
Data end & $2010-03$ & $2010-03$ & $2010-03$ & $2010-03$ & $2010-03$ & $2010-03$ \\
\hline
\end{tabular}

Notes. The table contains estimation results for the models:

Model 1: $\Delta \ln P_{s} \approx c+Y_{s} \Delta t-D_{t} \Delta Y_{s}$,

Model 2: $\Delta \ln P_{s} \approx c+\left(Y_{s}+\pi_{s}\right) \Delta t-D_{t} \Delta Y_{s}$,

Numbers in parentheses are the $t$-statistics for the estimated coefficients.

\section{References}

Y. Ait-Sahalia. Testing continuous-time models of the spot interest rate. Review of Financial Studies, 9(2):385-426, 1996.

E. Biffis and D. Blake. Securitizing and tranching longevity exposures. Insurance: Mathematics and Economics, 46(1):186 - 197, 2010.

D.M. Chance and J.V. Jordan. Duration, convexity, and time as components of bond returns. The Journal of Fixed Income, 6(2):88-96, 1996. 
P.O. Christensen and B.G. Sørensen. Duration, convexity, and time value. The Journal of Portfolio Management, 20(2):51-60, 1994.

M. Deacon, A. Derry, and D. Mirfendereski. Inflation-indexed Securities: Bonds, Swaps and Other Derivatives. John Wiley \& Sons, 2004.

F.J. Fabozzi. Bond Markets, Analysis, and Strategies. Prentice Hall, 2010.

F.J. Fabozzi. The Handbook of Fixed Income Securities. McGraw-Hill Education, 8th edition, 2011.

P. Hilli, M. Koivu, and T. Pennanen. Optimal construction of a fund of funds. European Actuarial Journal, 1:345-359, 2011a.

P. Hilli, M. Koivu, and T. Pennanen. Cash-flow based valuation of pension liabilities. European Actuarial Journal, 1:329-343, 2011 b.

A. Ilmanen. How well does duration measure interest rate risk? The Journal of Fixed Income, 1(4):43-51, 1992.

R. A. Jarrow. The relationship between yield, risk, and return of corporate bonds. The Journal of Finance, 33(4):12351240, 1978.

O. Kallenberg. Foundations of Modern Probability. Springer-Verlag, New Yor, NY, 2002.

F.K Reilly, W. Kao, and D.J. Wright. Alternative bond market indexes. Financial Analysts Journal, 48(3):44-58, 1992. 